\title{
Magnetic and Transport Properties of PdH: Intriguing Superconductive Observations*
}

\author{
Paolo Tripodi ${ }^{1 \dagger}$, Daniele Di Gioacchino ${ }^{2}$, and Jenny Darja Vinko ${ }^{1}$ \\ ${ }^{1}$ H.E.R.A., Hydrogen Energy Research Agency, Corso della Repubblica 448, 00049 Velletri, Italy \\ ${ }^{2}$ INFN-LNF National Institute of Nuclear Physics - National Laboratory of Frascati, Via Enrico Fermi 40, 00044 Frascati, Italy
}

Received on May 21, 2003

\begin{abstract}
Since the discovery of superconductivity in palladium-hydrogen $(\mathrm{PdH})$ and its isotopes $(\mathrm{D}, \mathrm{T})$ at low temperature, several efforts have been made to study the properties of this system. Superconductivity of PdH system has been initially claimed by resistance drop versus temperature and then confirmed by dc magnetic susceptibility measurements. These studies have shown that the critical transition temperature is a function of the hydrogen concentration $x$ in the $P d H_{x}$ system. In all these experiments, the highest concentration $x$ of hydrogen in palladium was lower than the unit. In the last decade we defined a room temperature and room pressure technique to load hydrogen and its isotopes into palladium at levels higher than unit, using electrochemical set-up, followed by a stabilization process to maintain the hydrogen concentration in palladium lattice stable. In the meanwhile, several measurements of resistance versus temperature have been performed. These measurements have shown several resistive drops in the range of $\left[18 \mathrm{~K}<T_{c}<273 \mathrm{~K}\right]$ similar to the results presented in literature, when the superconducting phase has been discovered. Moreover, on PdH wires $6 \mathrm{~cm}$ long the current-voltage characteristic with a current density greater than $6 * 10^{4} \mathrm{Acm}^{-2}$ has been measured at liquid nitrogen temperature. These measurements have the same behavior as superconducting I-V characteristic with sample resistivity, at $77 \mathrm{~K}$, of two orders of magnitude lower than copper or silver at the same temperature. The measurements of first and third harmonic of ac magnetic susceptibility in $P d H_{x}$ system have been performed. These represent a good tool to understand the vortex dynamics, since the superconducting response is strongly non-linear. Clear ac susceptibility signals confirming the literature data at low temperature $(9 \mathrm{~K})$ and new significant signals at high temperature (263K) have been detected. A phenomenological approach to describe the resistance behaviour of $\mathrm{PdH}$ versus stoichiometry $x$ at room temperature has been developed. The value $x=1.6$ to achieve a macroscopic superconducting state in $P d H_{x}$ has been predicted.
\end{abstract}

\section{Introduction}

\subsection{PdH $_{x}$ : overview}

Hydrogen $(\mathrm{H})$ and its isotopes dissolve in many metals and occupy interstitial sites in the host lattice producing an expansion of the lattice. Each interstitial $\mathrm{H}$ causes displacement of the metal atoms from their original sites while its electrons fill the electronic band of the metal at the Fermi energy level $E_{F}$. This gives rise to a series of changes in physical properties of the hosting metal $[1,2]$. In this framework Palladium $(\mathrm{Pd})$ is a metal, intensely studied, for much of its peculiarities [3]. Usually it is employed as catalyst for hydrogen reactions [4]. Generally, for Pd the $4 d^{10}$ state should be completely filled but in reality the 10 electrons are shared between two zones and the $E_{F}$ intersects the $4 \mathrm{~d}$ and the broad 5 sp bands $[3,5]$. There are 0.36 electrons in the $5 \mathrm{sp}$ band [5] and equal number of positive holes of unfilled state in $\mathrm{d}$ shell. For this reason Pd absorbs $\mathrm{H}$ very strongly. The paramagnetic susceptibility of pure Pd decreases from $550-600 * 10^{-6} \mathrm{cgs}$ units to diamagnetic behaviour of $P d H_{x}$ for stoichiometry $x=0.75$, where $x$ represents the ratio between $\mathrm{Pd}$ and $\mathrm{H}$ moles [4]. These measurements lead to deduction that the electron of the $\mathrm{H}$ goes into the $\mathrm{d}$ shell, thereby the number of positive holes decreases and hence the paramagnetic behaviour diminishes. There is a relationship between the electrical resistance of $P d H_{x}$ and the $\mathrm{H}$ stoichiometric value $x$ (Fig. 1). Analysing the relative resistance $R(x) / R_{o}$, where $R(x)$ is the $P d H_{x}$ electrical resistance versus $x$ and $R_{o}$ is the pure Pd electrical resistance, an experimental pseudo-parabolic curve with negative concavity is observed. At room temperature, this curve has a maximum at $x \approx 0.75$ and then it decreases almost to the initial value at $x \approx 1$. Considering that ideally in the fcc Pd lattice it should be possible to achieve a value $x=3$ when all the octahedral and tetrahedral sites are occupied, immediately rises the question: what happens to the electrical resistivity of the PdH at room temperature for $x>1$ ? For low temperatures, with $x \leq 1$ the answer is well known: $P d H_{x}$ system becomes superconductor $[7,8]$ with $T_{c}$ proportional to the $x$, namely, from $x=0.75$ with $T_{c}=1 \mathrm{~K}$ up to $T_{c}=9 \mathrm{~K}$ for $x$ close to 1 . We will attempt a reply to our questions through experimental details.

\footnotetext{
*Presented at the Workshop on Unconventional Superconductors (U-Super), Campinas May 20-24, 2003.

${ }^{\dagger}$ Also at INFN-LNF National Institute of Nuclear Physics National Laboratory of Frascati, Via Enrico Fermi 40, 00044 Frascati, Italy; paolo.tripodi@heraphysics.it
} 


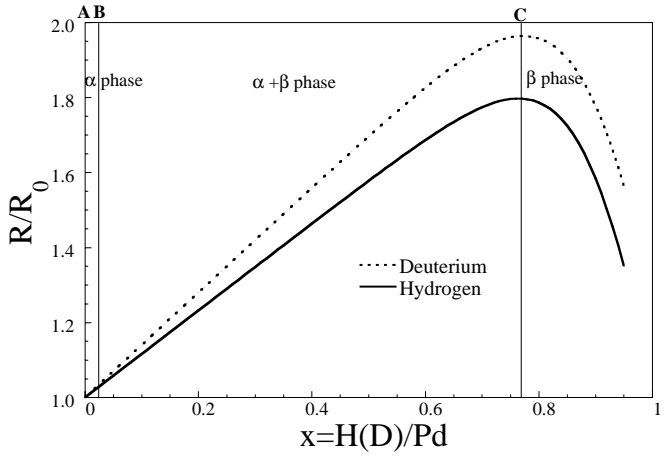

Figure 1. Relative resistance $R / R_{o}$ versus $\mathrm{H}$ stoichiometry $x=\mathrm{H} / \mathrm{Pd}\left(\mathrm{D} / \mathrm{Pd}\right.$ ) for $P d H_{x}$ sample (full line) and for $P d D_{x}$ (dotted line). $R_{o}$ is the resistance of pure Pd sample at stoichiometry $x=0$.

\section{2 $P d H_{x}$ : thermal coefficient of resistance $\lambda$}

We have studied the thermal coefficient of resistance $\lambda$ for $P d H_{x}$ system. It is well known that the resistance of metallic compounds depends on temperature. For a narrow temperature range, the resistance is described by equation (1):

$$
R(T)=R\left(T_{o}\right)(1+\lambda \Delta T)
$$

where $\mathrm{R}\left(T_{o}\right)$ is the resistance of the sample at $0^{\circ} \mathrm{C} . \Delta T=$ $T-T_{o}$ is the change in temperature and $\lambda$ represents the temperature coefficient of resistance. From the equation (1) the $\lambda$ equation (2) is calculated:

$$
\lambda=\left(\frac{R(T)}{R\left(T_{o}\right)-1}\right) * \Delta T^{-1}
$$

where $\lambda$ expresses the sensitivity of the resistive element versus temperature.

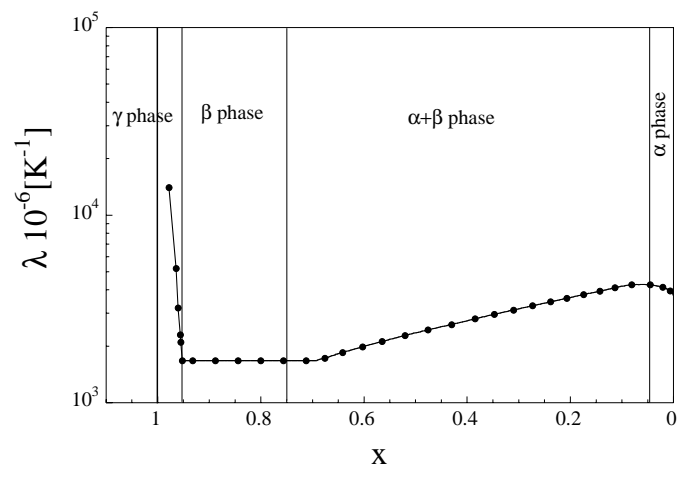

Figure 2. Thermal coefficient $\lambda$ of $P d H_{x}$, in function of hydrogen stoichiometry $x$ [6]. The horizontal axes has been reverted for a better comparison with Fig. 3 .

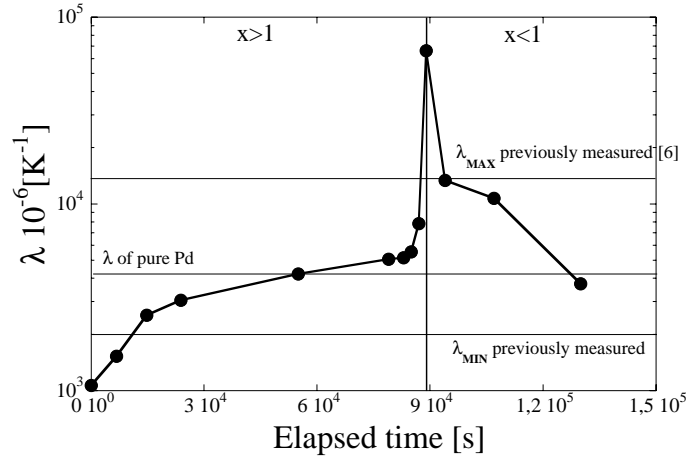

Figure 3. Thermal coefficient $\lambda$ of $P d H_{x}$, in function of time during a slight $\mathrm{H}$ deloading (Elapsed time). Each experimental point represents a measurement of $\lambda$ in the temperature range $273-373 \mathrm{~K}$. In these measurements the exact stoichiometry $x$, is not known, but $x>1$ is present at the beginning of the experiment, i.e. when the elapsed time $(\tau)$ is in the range $\left[0 \mathrm{~s}<\tau<9 * 10^{4} \mathrm{~s}\right]$. After the $\mathrm{H}$ loading the sample was stabilized and measured in temperature as to define $\lambda$ versus elapsed time while the sample slightly leaks $\mathrm{H}$.

There are contributions to the resistivity other than those due to lattice vibrations. For example, if a second element is present in a metal either as an impurity or as an alloying element, this destroys the periodicity in the electrical potential and gives rise to elastic scattering, hence the resistivity increases. On the other hand, if a short or long range order is present then the resistivity decreases. Clearly the resistivity and in particular $\lambda$, are rather sensitive to the order/disorder states [9]. It is well known that $\lambda$ is also dependent on the variation of stoichiometry $x$. Pure Pd (Fig. 2) has $\lambda=4000 p p m$ then it increases to a maximum of $\lambda=4200 \mathrm{ppm}$ at $x \approx 0.05$ corresponding to the phase transition between the $\alpha$ and the $\alpha+\beta$ phase. Increasing the $\mathrm{H}$ stoichiometry $x, \lambda$ decreases reaching a minimum of $\lambda=1800 \mathrm{ppm}$ at $x \approx 0.75$ corresponding to the phase transition between the $\alpha+\beta$ and the $\beta$ phase where $\lambda$ remains constant [4]. Then a steep increase of $\lambda=15000$ ppm (Fig. 2) has been measured at about $x \approx 1$ [6]. This quasi-asymptotic behaviour shown in Fig. 2 would indicate a possible new phase transition between the $\beta$ phase and the new $\gamma$ phase at $x=0.95[6]$.

In Fig. 3 we show further new experimental data demonstrating that with $x>1$ the coefficient $\lambda$ decreases to lower values, that confirm the presence of the phase transition between the $\beta$ and the new $\gamma$ phase [6]. Experimental method to obtain these results is as follows: in the temperature range between the ice melting temperature $T_{0}$ and the water boiling temperature $T_{B}$, for fixed stoichiometry $x$, the temperature coefficient of resistance $\lambda$ can be considered constant, i.e. we assume that in the temperature range $\Delta \mathrm{T}=T_{B}-T_{0}$ the slope of resistance versus temperature is constant as claimed in the formula (1). After the loading procedure the sample was cooled to $T_{0}$ and the resistance was measured. After that, the sample was warmed to $T_{B}$ and again the resistance was measured. Using the measured resistance and temperature values, $\lambda$ has been calculated with equation (2). In order to change the stoichiometry $x$ the sample was immersed 
again in the electrolitycal cell in floating configuration to obtain a slight $\mathrm{H}$ deloading. This procedure was repeated several times and in Fig. 3 every experimental point represents a measurement of $\lambda$ in function of the elapsed time during slow $\mathrm{H}$ deloading. During these measurements it was impossible to measure the H stoichiometry $x$ in the sample. The only available indication on $x$ is that the stoichiometry is decreasing in time, i.e at elapsed time zero, $x$ is maximum and then it decreases with time. The last three $\lambda$ points, in Fig. 3, are coherent with $\lambda$ values for $x<1$ shown in Fig. 2 [6]. This indicates that the other points in Fig. 3 show $\lambda$ at very high stoichiometry $x>1$. In conclusion, in the new $\gamma$ phase $(x>1)$ the $\lambda$ value is extremely low, in some cases we have measured a $\lambda$ as low as $1 \mathrm{ppm}$, whereas close to the transition stoichiometry $(x \approx 1) \lambda$ achieves very high values, up to $66000 \mathrm{ppm}$.

\section{3 $\mathrm{PdH}_{x}$ : electrical transport measurements}

A series of electrical transport measurements on $P d H_{x}$ wires, with $50 \mu \mathrm{m}$ diameter, will be presented. Experimental data on the $P d H_{x}$ behaviour at very high, unexplored, stable H concentration $x$ versus temperature (Fig. 4) shows a resistance drop at $T_{c}=51.6 \mathrm{~K}$. Applying $1 \mathrm{~T}$ dc magnetic field, the resistive drop splits to two lower temperatures of $31.3 \mathrm{~K}$ and $18.8 \mathrm{~K}[10,11]$. This data renders more consistent, but not definitive reply to our question, on the presence of a possible superconducting phase. In the light of this data, we prepared several $P d H_{x}$ samples with very high stoichiometry $x$ and hence several resistive drops have been measured from $80 \mathrm{~K}$ up to $273 \mathrm{~K}$ as shown in Fig. 4. These measured resistance drops, from $5 \%$ to $10 \%$ of the offset resistance values, have already been published $[10,11]$. The presence of these resistance drops for different temperatures $T_{c}$ indicates different $x$ concentrations in the samples and that only a small fractions of the sample have the $x$ value such as to cause the decreases of the resistance at given temperature. In order to calculate the corresponding $x_{c}$ values we used the equation presented in Fig. 13. This equation correlates $x_{c}$ with $T_{c}$. Another interesting measurement is the I-V characteristic for a $P d H_{x}$ sample at $L N_{2}$ temperature that shows superconducting critical current greater than $6 * 10^{4}$ $\mathrm{Acm}^{-2}$ (Fig. 5) [10]. The I-V value threshold is by 2 orders of magnitude higher, as we have already underlined in the reference [10]. However, our evaluation is based on the fact that the voltage drop on the $P d H_{x}$ wire at $77 \mathrm{~K}$ is a constant value with a variable current I. This cannot be a behavior of a normal resistance since there is no slope of the $\mathrm{I}-\mathrm{V}$ characteristic. Moreover, the rapid increase of the voltage drop $\mathrm{V}$ around the current value of $1 \mathrm{~A}$ indicates a rapid resistance increase. As shown in Fig. 5, the effective $P d H_{x}$ sample resistivity at $77 \mathrm{~K}$ is $5 \mathrm{n} \Omega \mathrm{cm}$ which is lower than copper and silver resistivity at $77 \mathrm{~K}$ respectively of $300 \mathrm{n} \Omega \mathrm{cm}$ and $200 \mathrm{n} \Omega \mathrm{cm}$.

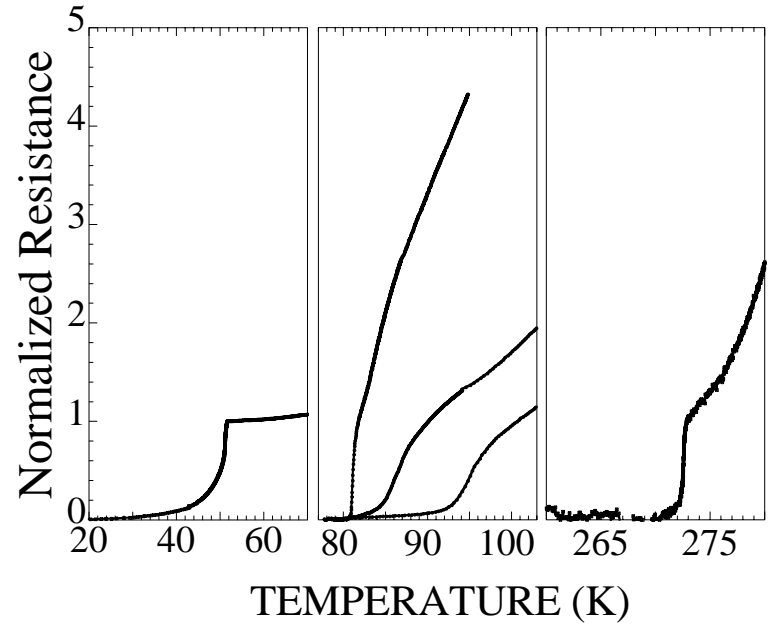

Figure 4. A series of superconducting transitions for $P d H_{x}$. The normalized resistance versus temperature is shown, as reported in literature [8]

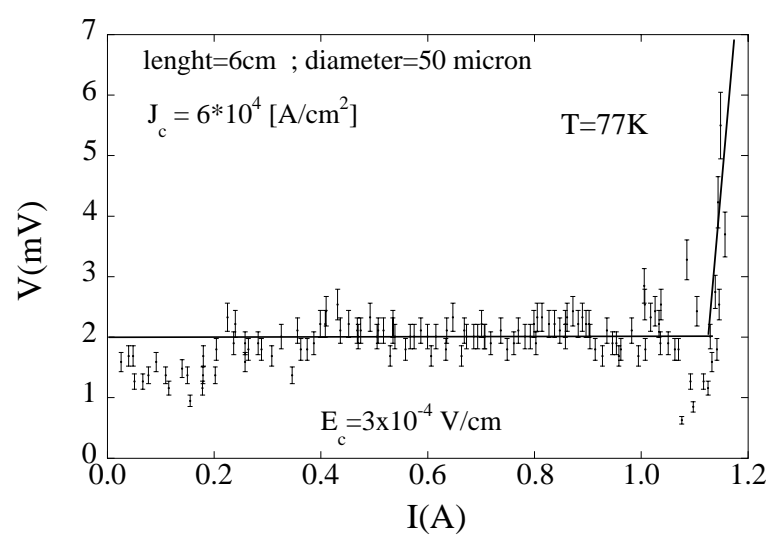

Figure 5. Critical current in $P d H_{x}$ wire at $\mathrm{T}=77 \mathrm{~K}$ [10].

\section{$1.4 \mathrm{PdH}_{x}$ : sample preparation}

$\mathrm{H}$ was loaded into the Pd lattice using an electrochemical cell. This chemical absorption process of $\mathrm{H}$ was used preferentially over physical absorption ( via $H_{2}$ gas pressure) for one primary reason. This method allows higher $\mathrm{H} / \mathrm{Pd}$ concentrations to be achieved at room temperature and atmospheric pressure in respect to other processes. The cell geometry consists of two parallel $\mathrm{Pt}$ square plates $\left(100 \times 100 \times 0.05 \mathrm{~mm}^{3}\right)$ as anode electrodes, separated by $15 \mathrm{~cm}$ of electrolyte. The cathode was a Pd sample, placed in the middle between the two anodes. The used Pd samples were thin wires $(50 \mu \mathrm{m}$ diameter, lenght in the range 6$30 \mathrm{~cm})$ or tapes $(50 \mu \mathrm{m}$ thickness, lenght in the range $2-4 \mathrm{~cm}$, width $0.4 \mathrm{~cm}$ ). This unusual geometry and a short loading time were adopted to maximize the $\mathrm{H}$ loading in $\mathrm{Pd}$ cathode and to minimize the transfer of anodically produced species 
to the cathode. The attainment of very high loading levels was found to be critically dependent on controlling the impurities in the electrolyte. The electrolyte consisted of strontium sulfate $\left(\mathrm{SrSO}_{4}\right)$ dissolved in $18 \mathrm{M} \Omega \mathrm{cm}$ water $\left(10^{-5} \mathrm{M}\right)$ giving a slightly acidic solution $(5.0 \leq \mathrm{pH} \leq 6.5)$. The electrolysis required a dc current from $5 \mathrm{~mA}$ up to $200 \mathrm{~mA}$. During electrolysis the four probe ac resistance measurements of the Pd cathode were taken using an RCL meter at $1 \mathrm{~mA}$, $1 \mathrm{KHz}$ sinusoidal current. Highly loaded $P d H_{x}$ samples, were electrochemically stabilised by adding $\left(10^{-5} \mathrm{M}\right)$ mercurous sulfate $\left(\mathrm{Hg}_{2} \mathrm{SO}_{4}\right)$ to the electrolyte. With this procedure the coated cathodes are found to be resistant to $\mathrm{H}$ deloading for periods of months at room temperature and pressure. The $\mathrm{Pd}-\mathrm{Hg}$ "alloy" forms an amalgam that covers the Pd surface and the depth of this "coating" is hundreds of nanometers, so that the effect of the resistance decrease, due to this coating, is negligible.

\section{5 $\operatorname{PdH}_{x}$ : mean stoichiometry measurement}

Important characterization of the sample is the measurement of the average final stoichiometric value $x$. In order to obtain the $x$ value, it is necessary to know the value of $\mathrm{H}$ and Pd moles. Pd moles are measurable with a sensitivity of about $1 \mu \mathrm{mol}$, but due to the low weight of $\mathrm{H}$ moles inside the Pd sample these measurements for hydrogen are not simple. For this purpose we have defined an electrochemical method, destructive however, for the $P d H_{x}$ sample. Therefore it can only be used as a standard method for the measurement of relative resistance $\mathrm{R}(x) / R_{o}$ versus $x$ as described below. To calculate $x$ we need to evaluate the efficiency $\varepsilon$ during the electrochemical $\mathrm{H}$ deloading process to avoid the undesired overestimation of $x$. $\varepsilon$ is defined as ratio between the variation of stoichiometry $\Delta x$ correlated with deloaded $\mathrm{H}$ from the sample and the corresponding amount of electrical charge $\Delta Q$ transferred through the electrodes:

$$
\varepsilon=\frac{\Delta x}{\Delta Q}
$$

For the $\varepsilon$ calculation the following experimental evidences are used:

1. the behavior of $\mathrm{R}(x) / R_{o}$ versus $x$ of the $P d H_{x}$ (fig.1) has 3 experimental constraints:

(a) point (A) where $\mathrm{R}(x) / R_{o}=1$ for $x=0$;

(b) point (B) that is the separation point between $\alpha$ and $\alpha+\beta$ phase where $\mathrm{R}(x) / R_{o}=1.07$ for $x \approx 0.05$;

(c) point $(\mathrm{C})$ the maximum of the curve where $\mathrm{R}(x) / R_{o}=1.76$ for $x=0.75$;

2. The electric charge $Q$ passed through the electrodes during the deloading of $P d H_{x}$ samples, proportional to the amount of $\mathrm{H}$ moles that exit Pd lattice.

For the average final value $x$, the following is used:

1. The final value $\mathrm{R}(x) / R_{o}$ achieved during the $\mathrm{H}$ loading in $\mathrm{Pd}$ (point D Fig. 6);
2. The estimated efficiency $\varepsilon$ value.

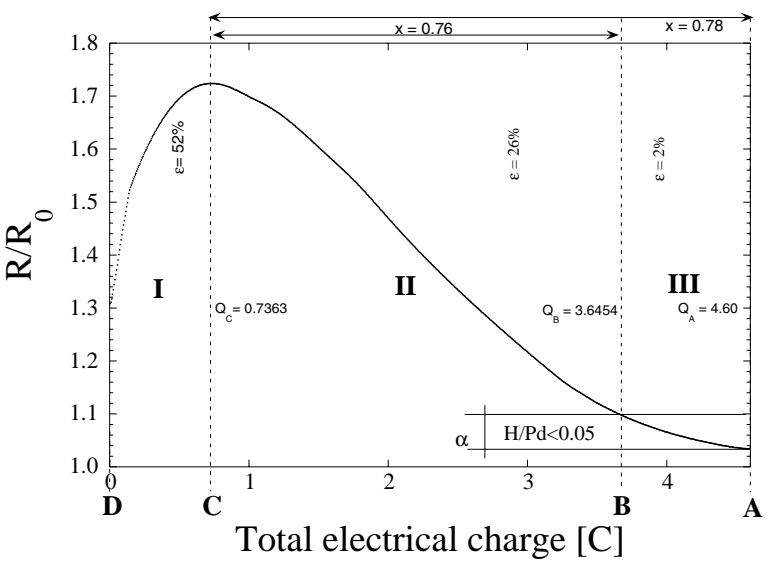

Figure 6. $\mathrm{R}(x) / R_{o}$ during $\mathrm{H}$ deloading versus electric charge passed trough the electrodes.

During the $\mathrm{H}$ loading, electrochemical concurrent processes and long loading time affect the efficiency calculation $\varepsilon$ (overestimation) particularly in the zone I (Fig. 6), so that it is necessary to calculate $\varepsilon$ during the $\mathrm{H}$ deloading. This way $\varepsilon$ is underestimated and hence renders acceptable $x$ value. In Fig. 6 the $\mathrm{R}(x) / R_{O}$ during the $H$ anodic deloading in $P d H_{x}$ wire sample is plotted in function of the total electric charge transferred through the electrodes. The behavior is divided in 3 regions: $\mathrm{I}$ in the interval [D-C], II in the interval $[\mathrm{C}-\mathrm{B}]$ and III in the interval [B-A]. To estimate the $\varepsilon$ value we start evaluating the separation point between the zone II and the zone III (point B) which fits the transition point between the $\alpha$ and $\alpha+\beta$ phase. The electric charge necessary to change the $\mathrm{R}(x) / R_{o}$ from the value 1 (point $\mathrm{A}$ ) to 1.07 (point $\mathrm{B}$ ) is $\Delta \mathrm{Q}=1$ Coulomb. Comparing this experimental data with the data in item 1(b) we find that the efficiency is $\varepsilon=5 \%$, which is way too low. The $\mathrm{H}$ stripping dynamics is dominated by two processes: the superficial recombination and the $\mathrm{H}$ diffusion from the inside to the surface of the $\mathrm{Pd}$. Since the $\varepsilon$ is determined only by the superficial recombination and in the zone III the diffusion effect is dominant, the underestimation of the $\varepsilon$ occurs in this zone. Therefore the zone II must be considered for a more accurate evaluation of $\varepsilon$. In the interval [C-B] the electric charge is $\Delta \mathrm{Q}=2.8$ Coulomb and comparing this data with the items 1 (b) and 1(c), we find an efficiency of $\varepsilon=26 \%$. In the zone I the sample is overloaded with H. Initially, around the point (D) the deloading efficiency is $\varepsilon=100 \%$ until the change of the slope (Fig. 6), to arrive to $\varepsilon=26 \%$ at the point (C). We presume that the average efficiency is $52 \%$ in this zone (doubled efficiency of the zone II) which is still strongly conservative estimation in respect to the arithmetic mean value $(\varepsilon=63 \%)$. 


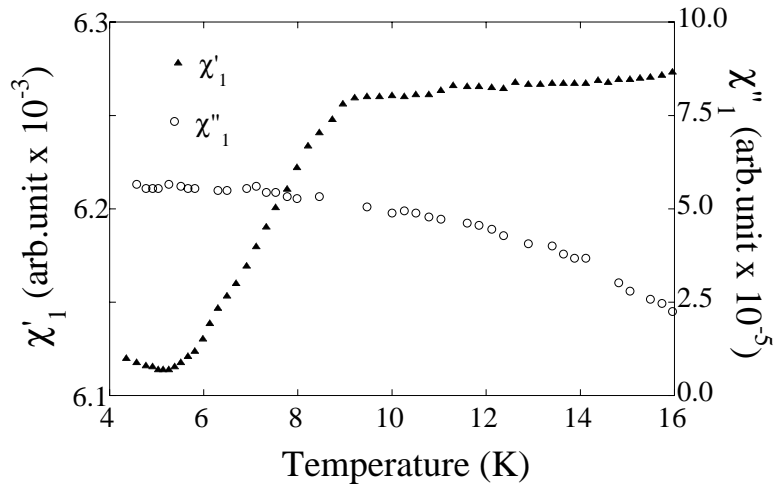

Figure 7. Real (triangles) and imaginary (circles) part of first harmonic $\left(H_{a c}=6 \mathrm{G} \mathrm{f}=1 \mathrm{KHz}\right)$ of $P d H_{x}$ slab $(\mathrm{x} \approx 1)$.

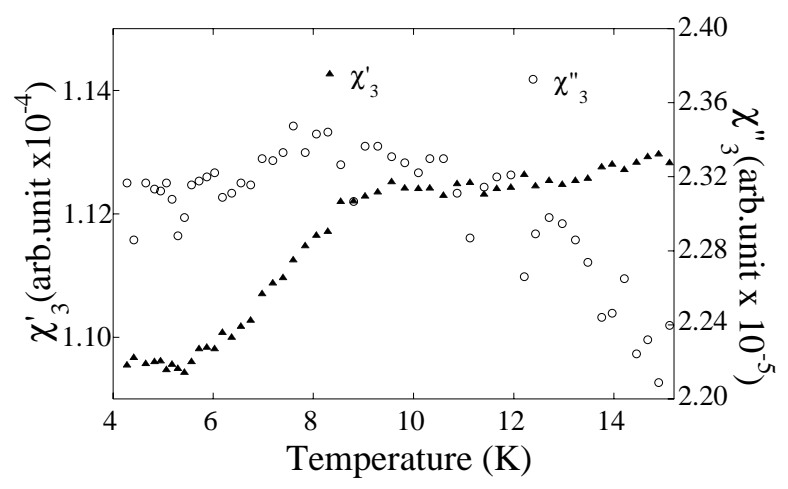

Figure 8 . Real (triangles) and imaginary (circles) part of third harmonic $\left(H_{a c}=6 \mathrm{G} \mathrm{f}=1 \mathrm{KHz}\right)$ of $P d H_{x}$ slab $(\mathrm{x} \approx 1)$.

From this definitive $\varepsilon$ value we obtain the final mean $x$ value. The electric charge in the zone I, from the point (C) to the point (D), is $\Delta \mathrm{Q}=0.8$ Coulomb. Hence the mole number of the $\mathrm{H}$ entered in the sample for the zone I, considering $\varepsilon=52 \%$, gives $\Delta x=0.4$. Now, as stated in item 1(c), the stoichiometry of the sample is $\Delta x=0.75$. Therefore the final stoichiometry is $x_{f} \geq 0.75+0.4=1.15$. Resuming this procedure, there are two important implications. First of all, this method is general and valid for any sample geometry. Secondly, it is independent from any concurrent electrochemical processes and from Pd sample moles.

\section{Results and Discussion}

To further define the possible superconducting properties of $P d H_{x}$ at high temperatures, we have performed and are presenting in this paper, the measurements of ac magnetic susceptibility and its higher harmonics. It is extremely important to check the presence of high harmonics since the superconducting response is strongly non-linear. In fact in the following section 2.1, we will discuss the first and third harmonic of the ac magnetic susceptibility measurements, moreover in section 2.2 a possible simple phenomenological approach that explains the $P d H_{x}$ relative resistance in function of $x$ at fixed temperature $(300 \mathrm{~K})$ will be presented.

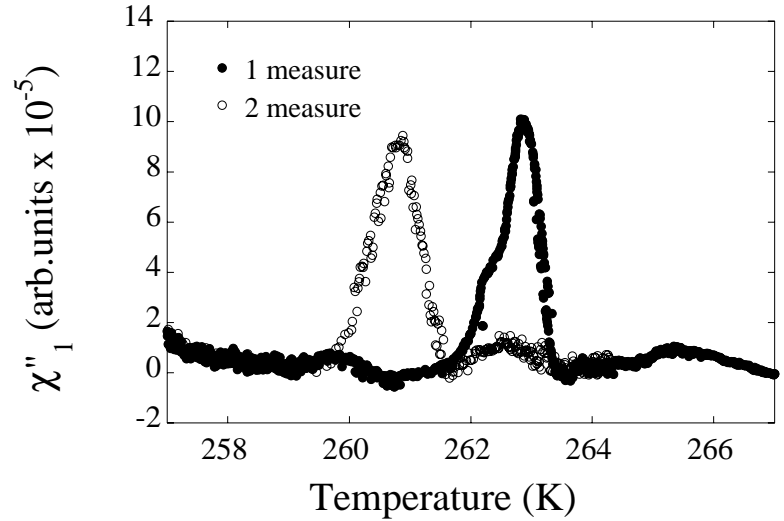

Figure 9. Imaginary parts of first harmonic of $P d H_{x}$ slab, first measure (full circles), second measure after 1 week (empty circles) $(x>1)$.

\section{1 ac susceptibility measurements}

The ac magnetic susceptibility $\left(\chi_{n}=\chi_{n}^{\prime}+\mathrm{i} \chi_{n}^{\prime \prime}: \mathrm{n}=1,3\right)$ measurements were performed with the European Facility 8T flow cryostat [12]. This cryostat has a susceptometer with an in-line double pick-up coil (Helmotz like) bridge with an exciting external ac magnetic field up to $6 \mathrm{G}$ at frequency in the range $[31 \mathrm{~Hz}<\mathrm{f}<2 \mathrm{kHz}]$, able to acquire with a lock-in amplifier the first seven harmonics of ac magnetic susceptibility, with the possibility to use a dc magnetic field up to 8T. The sample was cooled down to LHe temperature in ZFC (Zero Field Cooling) then the temperature has been slowly increased up to $300 \mathrm{~K}$. The data file collects the experimental value of ac susceptibility as the average value of 5 points while the temperature is not varied more than $0.01 \mathrm{~K}$. The subtraction of the null file due to the measurement system has been performed.

Some consideration on the phase measurement of the sample must be done for clarity. Usually in the ac magnetic susceptibility measurements the phase of the signal is imposed in order to maximize the in-phase $\left(\chi_{1}^{\prime}\right)$ component and minimize the out-phase $\left(\chi_{1}^{\prime \prime}\right)$ component when the sample is in superconducting or normal state (zero-phase procedure). In this case we minimized the imaginary part at LHe temperature. In Fig. 7 , the real $\chi_{1}^{\prime}$ and the imaginary $\chi_{1}$ components of the first harmonic are plotted at low temperature and a $T_{c}$ of $9 \mathrm{~K}$ [7] is shown. In Fig. 8, the third harmonic $\chi_{3}^{\prime}$ and $\chi_{3}^{\prime \prime}$ shows similar behavior of the sample. This indicates the presence of the well-known superconducting phase at low temperature. This superconducting phase, in accordance with literature data, is achieved for a stoichiometry $x \approx 1$ [7]. In Fig. 9 (first measurement) a very significant signal of the imaginary component $\chi_{1}^{\prime \prime}$ is shown at $263.5 \mathrm{~K}$, corresponding to zones of $P d H_{x}$ with stoichiometry $x>1$. A week later, the same measurements were repeated on this $P d H_{x}$ sample (second measurement), shown in Fig. 9, and the critical temperature shifted down 
to lower temperature $T_{c}=261.5 \mathrm{~K}$ (slight deloading, probably due to thermal cycles). A clear diamagnetic signal of the real component $\chi_{1}^{\prime}$ is shown in Fig. 10, overlapped with an extensive paramagnetic signal due to the $P d H_{x}$ zones with stoichiometry in the range $[0 \leq \mathrm{x}<0.75][4,13]$. This indicates that only a small fraction of the $P d H_{x}$ sample has the appropriate stoichiometry $x>1$ corresponding to the superconducting phase at this critical temperature $T_{c}=263.5 \mathrm{~K}$. This slope recalls the magnetic susceptibility behavior of the $\mathrm{RBa} \mathrm{Ba}_{2} \mathrm{Cu}_{3} \mathrm{O}_{7-x}$ with paramagnetic ordered rare earths $(\mathrm{R}=\mathrm{Sa}, \mathrm{Eu}$,etc. $)$ where, decreasing the temperature well below $T_{c}$, the behavior changes from diamagnetism to paramagnetism [14].

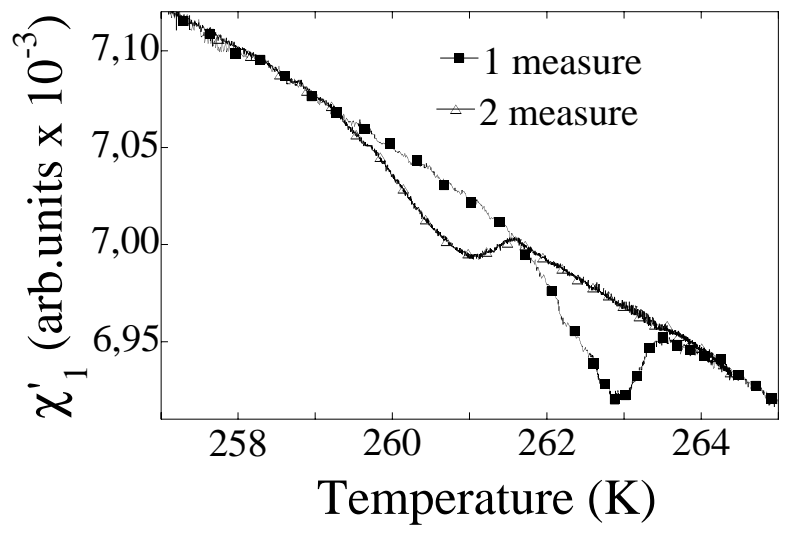

Figure 10. Real parts of first harmonic of $P d H_{x}$ slab, first measure (full squares), second measure after 1 week (empty triangles) $(x>1)$.

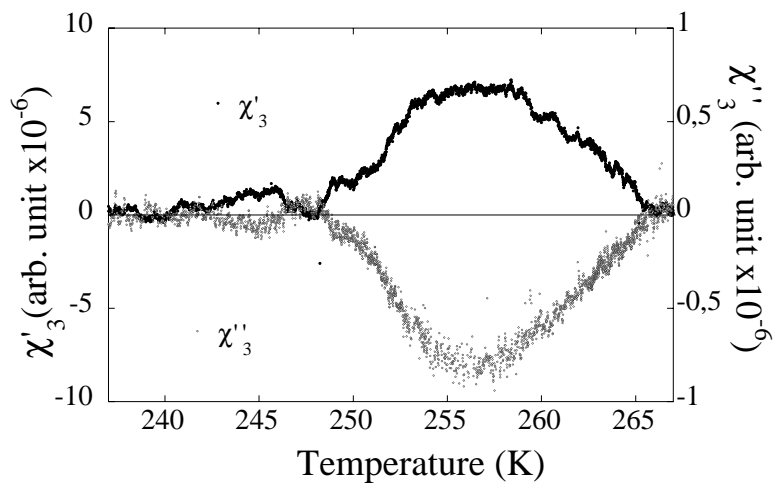

Figure 11. Real and imaginary parts of third harmonic of $P d H_{x}$ slab (first measure).

The fact that $\chi_{1}^{\prime}$ signal has a value lower than expected (about 1/6 less) in comparison with the imaginary component $\chi_{1}^{\prime \prime}$, can be imputed to the non-appropriate zero-phase procedure, in fact, the imaginary component has been minimized at $4.2 \mathrm{~K}$ and this procedure is strongly dependent on the temperature. We checked the presence of the third harmonic and in Fig. 11 the third harmonic real and imaginary part of first measure show a clear oscillation with an onset temperature of $264 \mathrm{~K}$. These results have been obtained after two months (room temperature and pressure) storage of these highly $\mathrm{H}$ loaded $P d H_{x}$ samples and the same sample rendered nearly the same $T_{c}$ in three consequent measurements.

\section{2 $R / R_{o}$ H loading at 300K: a phenomeno- logical approach}

The behaviour interpretation of the relative resistance $R / R_{o}$ at $300 \mathrm{~K}$ in function of $x$ content, uses the concept of the two phases $(\alpha, \beta)$ and their coexistence $(\alpha+\beta)$ in the $P d H_{x}$ system [3] but since $\mathrm{H}$ is not a parent metal of $\mathrm{Pd}$, this approach is not applicable. As shown in Fig. 12, a phenomenological model has been developed. The model predicts that $R / R_{o}$ of the $P d H_{x}$ systems at room temperature will exponentially decrease with the increasing of H loading $x$ for $x>1$. Moreover, it predicts that $R / R_{o}$ will decrease to zero resistance at $x_{c}=1.6$, so that the $P d H_{x}$ will be in a room temperature superconducting state. The $P d H_{x}$ system is modelled as having two electron transport mechanisms available in parallel: one superconducting fluctuation transport and the other normal transport. In other words, their corresponding resistances are in parallel to obtain the overall resistance.

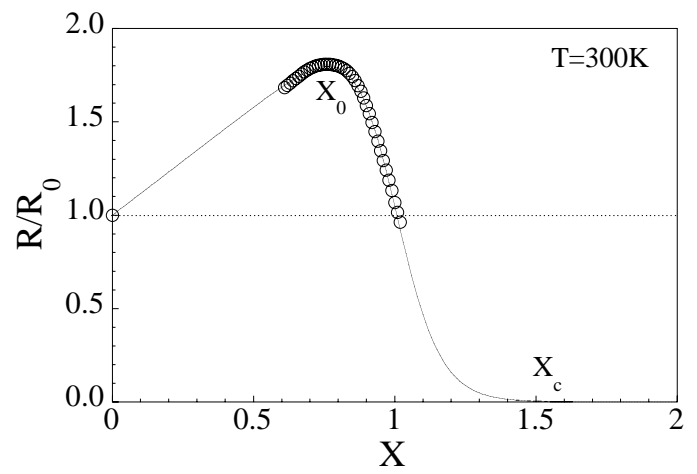

Figure 12. The best fit of experimental data for $P d H_{x}$ sample using the parallel model of the relative resistance. A critical temperature $T_{c}=300 \mathrm{~K}$ is achieved at $x_{c}=1.6$. The $x_{o}$ represents the $\mathrm{H}$ stoichiometry where the relative resistance is at maximum value.

The normal electron transport mechanism is modelled to describe a relative resistance that linearly increases with the stoichiometry $x$. This linear dependence is believed to result from the linear increase in the number of scattering centres in the system with the increase of $x$. Thus, the relative resistance is written as:

$$
\left(\frac{R}{R_{o}}\right)_{l}=1+\Lambda x
$$

where $\mathrm{R}$ is the resistance at stoichiometry $x, R_{o}$ is the resistance of a pure palladium and $\Lambda$ is a constant of proportionality. The superconducting fluctuation transport mechanism 
is modelled to describe a relative resistance that exponentially decreases with $x$. This exponential dependence for $x>1$ is written as:

$$
\left(\frac{R}{R_{o}}\right)_{e}=\beta e^{-\gamma\left(x-x_{o}\right)}
$$

where $R$ and $R_{o}$ are as defined in Eq. 4, $\beta$ is correlated with the frequency described in the superconducting fluctuation theory [15]. $\gamma$ is the ratio between the free energy increment [15] or the condensation energy of the superconducting domains and the Boltzmann factor $k T$ at $300 \mathrm{~K}$. $\left(x-x_{o}\right)$ represents the number of fluctuating superconductive domains for $x$ in the range $\left[0.75<x<x_{c}\right][16]$.

This superconducting fluctuation process can be explained as follows: During the $\mathrm{H}$ loading, the $\mathrm{H}$ electron fills the unfilled $4 \mathrm{~d}$ state easily without affecting much the $E_{F}$ level due to the high electron state density in 4 d shell. Increasing $\mathrm{H}$ stoichiometry, the electrons fill the unfilled 5sp bands above the $4 \mathrm{~d}$ bands. Due to the low electron state density of $5 \mathrm{sp}$ band, the $E_{F}$ must be raised. This leads us to deduce that the dissolved $\mathrm{H}$ atoms in $\mathrm{Pd}$ are completely ionised $\left(\mathrm{H}^{+}\right)$in interstitial position without being specifically bound to a particular Pd atom. The dissolved $\mathrm{H}^{+}$ions [5] are also extremely mobile. The transition rate is $10^{12}$ $\mathrm{s}^{-1}$ and the self-diffusion coefficient is $10^{-6} \mathrm{~cm}^{2} \mathrm{~s}^{-1} . \mathrm{H}^{+}$ ions localized in a cluster, in average remain about their sites for 10 oscillations before moving to nearest-neighbour sites. Meanwhile, the sub-lattice vibrates with very high localized optical frequencies at about $10^{13} \mathrm{~Hz}$, due to the lightness of the $\mathrm{H}$ mass. In this context a local fluctuation of superconductivity in $P d H_{x}$ can occur with a rather unusual coupling between localised optical phonons and the $E_{F}$ electrons that spent considerable time around $H^{+}$ions. This combination allows the electrons at $E_{F}$ level to experience the attraction through $\mathrm{H}$-sub-lattice (BCS like approach). Increasing $\mathrm{H}$ content, the number of clusters and local superconducting fluctuations increases achieving the complete superconducting phase.

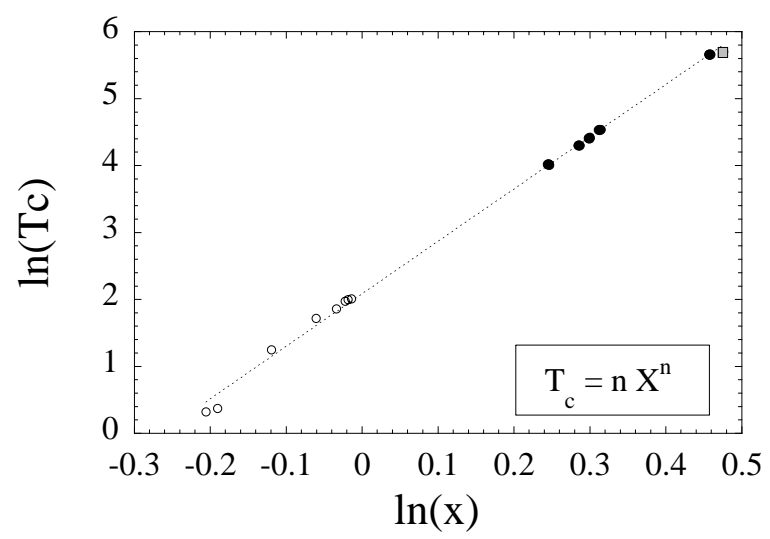

Figure 13. Experimental stoichiometry $x$ data for $P d H_{x}$ samples with low $T_{c}$ [7] (empty circles) together with our phenomenological approach datum (gray square). In accordance with this model, $x$ values for a series of experimental $T_{c}$ shown in Fig. 4 , have been printed as full circles.
Adding the relative resistances from Eq. 4 and Eq. 5 in parallel, one obtains a phenomenological expression for $R / R_{o}$ at room temperature as a function of $x$ :

$$
\left(\frac{R}{R_{o}}\right)_{p}=\frac{(1+\Lambda x) \beta e^{-\gamma\left(x-x_{o}\right)}}{1+\Lambda x+\beta e^{-\gamma\left(x-x_{o}\right)}}
$$

Using the fitted free parameters, the resulting curve is shown in Fig. 12. The best fit of equation (6) produces the final equations $(7,8)$ :

$$
\begin{gathered}
\left(\frac{R}{R_{o}}\right)_{l}=1+1.16 x \\
\left(\frac{R}{R_{o}}\right)_{e}=40 e^{-12.4(x-0.76)}
\end{gathered}
$$

In equation (7), the parameter $\Lambda=1.16$ is strictly correlated with the linear lattice expansion during $\mathrm{H}$ loading [1]. From equation (8) the condensation energy of $270 \mathrm{meV}$ for the superconducting phase can be calculated at $x=x_{c}$. This value is in the expected range for the formation energy of coherent systems. The equations $6-8$ render the value $R=0$ for $x_{c}=1.6$. This indicates a possible superconducting state at $300 \mathrm{~K}$ for $x_{c}=1.6$. This datum (gray square in Fig. 13) together with the experimental data at low $T_{c}$ (empty circles in Fig. 13) reported in literature, are used to extrapolate the relationship in Fig. 13: $T_{c}=\mathrm{n} x^{n}$. For $P d H_{x}$ system results $n=7.86$. Using this relationship we added the resistance drops data (Fig. 4) to the plot in Fig. 13 (full circles) to obtain a quantification of the mean stoichiometry $x$ that results in the range [1.27 $\left.<x_{c}<1.57\right]$.

\section{Summary and conclusions}

This letter presents first and third harmonics of ac susceptibility data together with our previous variety of experimental data [10] on electrical transport. These measurements together with several resistive drops in the temperature range $\left[51.6 \mathrm{~K}<T_{c}<273 \mathrm{~K}\right]$ give some further indication to answer the question: what happens at room temperature for $x>1$ in $P d H_{x}$ system? Should the $P d H_{x}$ system with $x>1$ be a HTSC, the presented data must be confirmed with a clear Meissner effect measurement. Nevertheless, a small diamagnetic signal of $\chi_{1}^{\prime}$ at $263 \mathrm{~K}$ in coincidence with imaginary part of $\chi_{1}^{\prime \prime}$ and real and imaginary part of third harmonics have been measured. A simple phenomenological approach based on the parallel of two electric transport mechanisms in order to explain the $R / R_{o}$ behaviour at room temperature in the $P d H_{x}$ system as a function of the $\mathrm{H}$ concentration $x$ has been developed. The first mechanism explains the experimental increases of the relative resistance in the $P d H_{x}$ system due to the lattice expansion in the range $\left[0<x<x_{o}=0.75\right]$. The second mechanism explains the decreases of the $P d H_{x}$ relative resistance at $x>x_{o}$, as a typical expression of the superconducting fluctuations of resistance. The superconducting state at room temperature $T_{c}=300 \mathrm{~K}$ for concentration $x_{c}=1.6$ has been predicted. 


\section{Acknowledgements}

We wish to thank our collaborators Antonio Celluci and Riccardo Romanato for their valuable collaboration.

\section{References}

[1] G. Alefeld and J.Völkl, Hydrogen in Metals I, SpringerVerlag (1978)

[2] G. Alefeld and J.Völkl, Hydrogen in Metals II, SpringerVerlag (1978)

[3] N.F. Mott and H.Jones, The Theory of the Properties of Metal and alloys, Dover Publications, Inc. (1936) 183,186

[4] F.A. Lewis, The Palladium Hydrogen, Academic Press (1967)

[5] M.H. Lee, Separation Science and Technology, 15, 457 (1980)

[6] P. Tripodi, M.C.H. McKubre, F.L. Tanzella, P.A. Honnor, D. Di Gioacchino, et.al, Physics Letters A 276, 122 (2000)

[7] T.Skoskiewicz, Phys. Stat. Sol. (a) 11, K123 (1972)

[8] B. Stritker, W. Buckel, Z. Physik 257, 1 (1972)
[9] R.J. Weiss, Solid State Physics for Metallurgists, Pergamon, Press. (1963) 235

[10] P. Tripodi, D. Di Gioacchino, R. Borelli, J.D. Vinko, Physica C 389, 571 (2003)

[11] Paolo Tripodi, patents WO0167525, EP1269550, AU7436301, US2003144151

[12] This susceptometer is located in Frascati National Laboratory (I.N.F.N.). Facility of Transnational Access to Research Infrastructure (TARI) contract HPRI-CT-1999-00088

[13] C. Kittel, Introduction to Solid State Physics $6^{\text {th }}$ editions Wiley\&Sons (1986) p.417

[14] H. Zhou, C. L. Seaman Y. Dalichaouch, B.W. Lee, K.N. Yang,R.R. Hake, M.B. Maple,R.P. Guertin, M.V. Kuric, Physica C 152, 321 (1998)

[15] M. Tinkham, Introduction to Superconductivity, McGrawHill (1996) 290-291

[16] P. Tripodi, D. Di Gioacchino, J.D. Vinko, Superconductivity in $\mathrm{PdH}$ : phenomenological explanation, Physica $\mathrm{C}$ in press. 